\title{
Difference in Psychological Distress between Patients with Periodic Limb Movement Disorder and Restless Legs Syndrome
}

\author{
Jung-Ick Byun, $M D^{1 *}$, Jeong-Su Kim, $\mathrm{MD}^{1 *}$, Yu Yong Shin, $\mathrm{MD}^{1}$, Kyoung Jin Hwang, MD, PhD², \\ Yu Jin Jung, MD, $\mathrm{PhD}^{3}$, Won Chul Shin, MD, $\mathrm{PhD}^{1,2}$ \\ ${ }^{1}$ Department of Neurology, Kyung Hee University Hospital at Gangdong, Seoul, Korea \\ 2Department of Neurology, School of Medicine, Kyung Hee University, Seoul, Korea \\ ${ }^{3}$ Department of Neurology, Daejeon St. Mary's Hospital, College of Medicine, The Catholic University of Korea, Daejeon, Korea
}

Received: February 18, 2019

Revised: April 24, 2019

Accepted: May 2, 2019

Correspondence

Won Chul Shin, MD, PhD

Department of Neurology,

Kyung Hee University Hospital at Gangdong,

892 Dongnam-ro, Gangdong-gu,

Seoul 05278, Korea

Tel $+82-2-440-6166$

Fax +82-2-440-7262

E-mailshin1chul@gmail.com

*These authors contributed equally to this work.

ORCID

Jung-Ick Byun

https://orcid.org/0000-0002-6224-4575

Jeong-Su Kim

https://orcid.org/0000-0002-5560-0201

Yu Yong Shin

https://orcid.org/0000-0002-0032-1096

Kyoung Jin Hwang

https://orcid.org/0000-0003-0856-2203

Yu Jin Jung

https://orcid.org/0000-0003-4643-1968

Won Chul Shin

https://orcid.org/0000-0003-3044-9397
Background and Objective We used the Symptom Checklist 90-Revised (SCL-90-R) to compare the psychosomatic symptom profiles between periodic limb movement disorder (PLMD) and restless legs syndrome (RLS) patients.

Methods Twenty consecutive drug-naïve RLS patients, 20 age- and sex-matched patients with PLMD and healthy controls were enrolled. Questionnaires evaluating sleep (Pittsburgh Sleep Quality Index, and Insomnia Severity Index), depression (Beck Depression Inventory II), and psychosomatic symptoms (SCL-90-R) were administered. Polysomnography was performed in the patients with PLMD or RLS.

Results The mean age of the subjects was $57.5 \pm 10.9$ years, and half of the subjects were male. Compared with the patients with RLS, the patients with PLMD exhibited a reduced total sleep time $(276.6 \pm 41.3$ vs. $322.4 \pm 42.9, \mathrm{p}=0.04)$ and a decreased proportion of REM sleep (12.5 \pm 6.6 vs. $17.8 \pm 7.8, \mathrm{p}=0.009)$. The patients with RLS exhibited higher SCL-90-R T-scores on the somatization (post hoc $\mathrm{p}<0.001$ ), obsessive-compulsive (post hoc $\mathrm{p}<0.001$ ), interpersonal sensitivity (post hoc $\mathrm{p}=0.003$ ), anxiety (post hoc $\mathrm{p}=0.005$ ), and psychoticism (post hoc $\mathrm{p}=0.004$ ) domains than the controls. Compared to the controls, the patients with PLMD exhibited higher SCL-90-R scores only in hostility (post hoc $\mathrm{p}=0.016$ ). Somatization in the RLS patients was significantly worse than that in the PLMD patients (post hoc $\mathrm{p}=0.003$ ).

Conclusions The results of our study demonstrate that psychosomatic distress is greater in RLS patients and lesser in PLMD patients. Somatization was a distinguishing symptom between RLS and PLMD.

Sleep Med Res 2019;10(1):25-30

Key Words Periodic limb movement disorder, Restless legs syndrome, Psychosomatic symptoms, SCL-90-R.

\section{INTRODUCTION}

Restless legs syndrome (RLS) is a sensorimotor disorder with a diagnosis based on patient history and does not require polysomnography (PSG). The diagnostic criteria for RLS include 1) the urge to move the legs, 2) symptoms that worsen when lying down or sitting, 3) symptoms that are at least partially relieved by movement, and 4) symptoms that worsen in the evening or at night [1-3]. In contrast, the diagnosis of periodic limb movement disorder (PLMD) is mainly based on PSG results and symptoms of sleep disturbance [2]. The two disorders are distinct but related. More than $80 \%$ of RLS patients have periodic limb movements during sleep (PLMS) comparable to those with PLMD [4]. Both disorders respond to dopamine agonist treatment [5], suggesting that they share a common pathophysiology related to the dopaminergic pathway. Moreover, a recent report suggested common SNP loci between PLMD 
and RLS [6].

Mood disorders, including depression and anxiety, are common comorbidities of RLS [7]. Large community-based studies have reported that the lifetime prevalence of such mood disorders is elevated by 2.1 to 5.3 -fold in patients with RLS compared to that in controls [8,9]. In addition, panic disorders [8] are reportedly more common in RLS patients. PLMD is also known to be associated with depression and anxiety disorder [10]. A recent study reported similar degrees of sleepiness, depression and anxiety between patients with PLMD and those with RLS [11].

The role of PLMS in psychosomatic symptoms in patients with PLMD or RLS remains unclear [12]. PLMS may disrupt sleep and cause psychosomatic distress [13]; however, previous studies investigating the clinical significance of PLM have reported contradicting results [14]. Among RLS patients, PLMS was not associated with subjective sleep-related symptoms. Moreover, RLS patients without PLMS exhibited even worse depression and anxiety than those with PLMS [15].

The Symptom Checklist 90-Revised (SCL-90-R) is a structured self-reported questionnaire used to evaluate psychopathology and psychological distress. A few studies have evaluated psychosomatic distress in RLS by using the SCL-90-R. Patients with RLS report greater distress related to not only depression and anxiety but also somatization, compulsivity, and hostility. The SCL-90-R score has been shown to be associated with the severity of RLS and its treatment [16,17]. However, no study has evaluated the SCL-90-R score in patients with PLMD.

This study aimed to compare psychosomatic symptoms between PLMD and RLS patients and elucidate the association with PLM. We used the SCL-90-R to evaluate the psychosomatic symptoms in PLMD and RLS patients and assessed the association between the SCL-90-R domain scores and the PLM Index (PLMI).

\section{METHODS}

\section{Participants}

This retrospective cross-sectional study was performed at the Kyung Hee University Hospital at Gangdong sleep clinic. Previously, we performed PSG on consecutive drug-naïve patients with a clinical diagnosis of RLS between January 2007 and June 2008. Age- and sex-matched PLMD patients who did not meet the clinical criteria for RLS were selected from among those who visited our clinic for sleep disturbance and underwent PSG between January 2007 and December 2016. RLS was diagnosed by a sleep specialist based on the criteria proposed by the International RLS Study Group [18]. PLMD was diagnosed based on the PSG results (more than 15 periodic limb movements per hour of sleep) and clinical symptoms of insomnia or daytime sleepiness without any other sleep disorders. Individuals who are on medication for the RLS and PLMD, who were diagnosed as secondary RLS or PLMD due to other illnesses, and those with 1) comorbid obstructive sleep apnea (AHI $>5 / h r), 2$ ) other sleep disorder (i.e., REM sleep behavior disorder), 3) other neurological or psychiatric disorders, or 4) severe medical illness were excluded. Age- and sex-matched good-sleeper controls [Pittsburgh Sleep Quality Index (PSQI), score $<5$ ] were recruited from the same region as the patients. The controls were screened for sleep-related symptoms and neurological or psychological diseases using a structured questionnaire and a clinical interview.

This study was approved by the Institutional Review Board of the KyungHee University Hospital at Gangdong (IRB No. PMS2013-039-016). Written informed consent to participate was obtained from all enrolled patients.

\section{Procedures}

The participants completed questionnaires evaluating sleep [PSQI [19]; Epworth Sleepiness Scale (ESS) [20]; and Insomnia Severity Index (ISI) [21]], depression [Beck Depression Inventory II (BDI-II) [22]] and neuropsychiatric symptoms (SCL-90-R). The PSQI measures sleep quality and patterns over a 4-week period [19], the ESS is an 8-item self-reported questionnaire evaluating the level of daytime sleepiness [20], the ISI is a 7-item questionnaire measuring the patient's perceived insomnia [21], and the BDI-II measures depression with 21 multiple-choice questions, each of which can be scored from 0 to 3 [22]. The SCL-90-R is a self-rating questionnaire that evaluates psychopathology and psychological distress. The patient's symptoms are assigned to the following 9 categories: somatization (SOM), obsession-compulsion (O-C), interpersonal sensitivity (I-S), depression (DEP), anxiety (ANX), hostility (HOS), phobic anxiety (PHOB), paranoid ideation (PAR), and psychoticism (PSY). The overall psychological symptoms were measured using the following three global indexes: Global Severity Index (GSI), Positive Symptom Distress Index (PSDI), and Positive Symptoms Total (PST). The standard Korean version of the SCL-90-R [23] was used, and the results of the questionnaire are presented as T-scores with a mean of 50 and a standard deviation of 10 .

The PSG results of the RLS and PLMD patients were reviewed. PSG was performed according to standard protocols using a digital polygraph system (Grass Beehive ${ }^{\mathrm{TM}}$ Horizon, West Warwick, RI, USA). We recorded the data from six EEG channels (C3-A2, C4-A1, F3-A2, F4-A1, O3-A2, and O2-A1) according to the standard 10-20 system in addition to two electrooculography channels and surface electromyography of the chin and both anterior tibialis muscles. The data were scored manually according to the American Academy of Sleep Medicine (AASM) Manual for the Scoring of Sleep and Associated Events, Version 2.4 (AASM, 2017). We obtained data, including the total sleep time (TST); percentages of time spent in N1, N2, N3, and REM sleep; sleep latency; sleep efficiency; arousal index; apnea-hypopnea index; minimum saturation; periodic limb 
movement arousal index (PLMAI); and periodic limb movement indexes (PLMI).

\section{Statistical Analysis}

The continuous data were tested for normal distribution with the Kolmogorov-Smirnov test and are presented as the mean \pm $\mathrm{SD}$. Because the questionnaire score variables were nonnormally distributed, the Kruskal-Wallis $\mathrm{H}$ test was used for the between-group comparisons among the 3 groups (controls, RLS and PLMD), and the Mann-Whitney $U$ test was used for the post hoc pairwise comparisons. The PSG data of the RLS and PLMD groups were compared with the Mann-Whitney U test or t-test as appropriate. The nominal data were analyzed using the chi-square test. The correlations between the PLMI or PLMAI and the PSG variables, sleep questionnaires, and SCL-90-R Tscores were tested using the Spearman correlation after adjusting for age. The significance level was set to 0.05 , and for the post hoc analysis, the level of significance was set at $\mathrm{p} \leq 0.05 / 3$ (0.017). All statistical comparisons were conducted using SPSS (version 22.0, IBM Corp., Armonk, NY, USA).

\section{RESULTS}

\section{Patient Characteristics and PSG Results}

Twenty consecutive patients with RLS, age- and sex- matched patients with PLMD, and good sleeper controls were enrolled. The participants' mean age was $57.5 \pm 10.9$, and half of the participants were males. The mean BMI was $24.9 \pm 2.9 \mathrm{~kg} / \mathrm{m}^{2}$.

Compared with the RLS patients, the patients with PLMD showed a reduced TST ( $276.6 \pm 41.3$ vs. $322.4 \pm 42.9, \mathrm{p}=0.04)$, a decreased proportion of REM sleep ( $12.5 \pm 6.6 \mathrm{vs.} 17.8 \pm 7.8$, $\mathrm{p}=0.009)$, and a tendency toward reduced sleep efficacy $(74.2$ \pm 9.4 vs. $80.0 \pm 11.6, \mathrm{p}=0.066)$. The PLMI and PLMAI results of the RLS and PLMD patients were similar ( $\mathrm{p}=0.304$ and 0.372 , respectively) (Table 1 ). After adjusting for age, there was a significant correlation between the PLMI and the arousal in$\operatorname{dex}(r=0.372, p=0.02)$ and between the PLMAI and the proportion of $\mathrm{N} 1(\mathrm{r}=0.721, \mathrm{p}<0.001), \mathrm{N} 3(\mathrm{r}=-0.59, \mathrm{p}<0.001)$, and the arousal index $(\mathrm{r}=0.848, \mathrm{p}<0.001)$.

\section{Sleep Questionnaire Score}

The PSQI and ISI scores of the patients with PLMD (post hoc $\mathrm{p}<0.001$ each) or RLS (post hoc $\mathrm{p}<0.001$ each) were higher than those of the controls. There was no significant difference in the ESS and BDI-II scores among the three groups. No significant difference in the sleep questionnaire scores was found between the RLS and PLMD patients (Table 2). The PLMI and PLMAI were not correlated with any sleep questionnaire scores.

\section{SCL-90-R Scores}

Group differences were found in all SCL-90-R domains, ex- cept for PAR. The post hoc analysis revealed that the RLS patients had significantly higher SOM (post hoc $\mathrm{p}<0.001$ ), O-C (post hoc $\mathrm{p}<0.001$ ), I-S (post hoc $\mathrm{p}=0.003$ ), ANX (post hoc $\mathrm{p}=0.005$ ), and PSY (post hoc $\mathrm{p}=0.004$ ) domain scores than the controls. A tendency toward a higher score was found in DEP (post hoc $\mathrm{p}=0.018$ ), HOS (post hoc $=0.022$ ), and $\mathrm{PHOB}$ (post hoc $=0.017$ ). Additionally, the RLS patients had higher scores in the SOM domain than the patients with PLMD (56.9

Table 1. Demographics and polysomnography results

\begin{tabular}{lcccc}
\hline & $\begin{array}{c}\text { Controls } \\
\mathrm{n}=20\end{array}$ & $\begin{array}{c}\text { PLMD } \\
\mathrm{n}=20\end{array}$ & $\begin{array}{c}\text { RLS } \\
\mathrm{n}=20\end{array}$ & p-value* \\
\hline Age & $57.9 \pm 6.7$ & $55.0 \pm 12.0$ & $59.5 \pm 13.0$ & 0.386 \\
Sex $(\mathrm{male})$ & $15(50.0)$ & $13(65.0)$ & $8(40.0)$ & 0.280 \\
BMI $\left(\mathrm{kg} / \mathrm{m}^{2}\right)$ & $23.9 \pm 2.3$ & $25.8 \pm 3.1$ & $25.1 \pm 3.0$ & 0.080 \\
PSG & & & & \\
TST (min) & NA & $276.6 \pm 41.3$ & $322.4 \pm 42.9$ & 0.040 \\
N1\% & NA & $19.2 \pm 9.7$ & $20.3 \pm 16.0$ & 0.935 \\
N2\% & NA & $38.2 \pm 11.4$ & $33.9 \pm 11.1$ & 0.358 \\
N3\% & NA & $30.0 \pm 11.3$ & $27.6 \pm 12.1$ & 0.705 \\
R\% & NA & $12.5 \pm 6.6$ & $17.8 \pm 7.8$ & 0.009 \\
WASO \% & NA & $21.2 \pm 8.8$ & $19.2 \pm 11.4$ & 0.417 \\
SL (min) & NA & $26.0 \pm 39.7$ & $9.1 \pm 5.7$ & 0.151 \\
SE\% & NA & $74.2 \pm 9.4$ & $80.0 \pm 11.6$ & 0.066 \\
AI (/hr) & NA & $39.2 \pm 12.6$ & $39.3 \pm 27.3$ & 0.239 \\
AHI (/hr) & NA & $2.2 \pm 2.0$ & $1.9 \pm 2.5$ & 0.377 \\
Min sat\% & NA & $88.5 \pm 7.1$ & $90.3 \pm 3.6$ & 0.645 \\
PLMAI (/hr) & NA & $13.8 \pm 11.4$ & $22.5 \pm 29.4$ & 0.304 \\
PLMI (/hr) & NA & $41.0 \pm 22.9$ & $60.4 \pm 51.6$ & 0.372 \\
\hline
\end{tabular}

The data are expressed as the mean \pm standard deviation.

*Kruskal-Wallis H test or Mann-Whitney U test.

PLMD: periodic limb movement disorder, RLS: restless legs syndrome, BMI: body mass index, PSG: polysomnography, TST: total sleep time, WASO: waking after sleep onset, SL: sleep latency, SE: sleep efficacy, AI: arousal index, AHI: apnea-hypopnea index, PLMAI: periodic limb movement arousal index, PLMI: periodic limb movement index, NA: not available.

Table 2. Sleep questionnaire results

\begin{tabular}{lcccc}
\hline Questionnaire & $\begin{array}{c}\text { Controls } \\
\mathrm{n}=20\end{array}$ & $\begin{array}{c}\text { PLMD } \\
\mathrm{n}=20\end{array}$ & $\begin{array}{c}\text { RLS } \\
\mathrm{n}=20\end{array}$ & p-value* \\
\hline PSQI & $3.6 \pm 1.1$ & $8.8 \pm 4.3^{\dagger}$ & $11.7 \pm 3.9^{\dagger}$ & $<0.001$ \\
ISI & $4.5 \pm 3.6$ & $12.4 \pm 6.9^{\dagger}$ & $15.6 \pm 7.7^{\dagger}$ & $<0.001$ \\
ESS & $5.2 \pm 1.8$ & $5.9 \pm 4.2$ & $5.8 \pm 3.8$ & 0.706 \\
BDI-II & $9.8 \pm 5.4$ & $12.5 \pm 6.6$ & $16.2 \pm 8.9$ & 0.059
\end{tabular}

The data are expressed as the mean \pm standard deviation.

*Kruskal-Wallis H test or Mann-Whitney U test. ${ }^{\dagger}$ post hoc MannWhitney $\mathrm{U}$ test $\mathrm{p}<0.017$ compared with controls.

PLMD: periodic limb movement disorder, RLS: restless legs syndrome, PSQI: pittsburgh sleep quality index, ISI: insomnia severity index, ESS: epworth sleepiness scale, BDI-II: beck depression inventory II. 
\pm 12.0 vs. $47.4 \pm 7.8$, post hoc $\mathrm{p}=0.003$ ). Compared with the controls, the PLMD patients showed a significant increase only in the HOS (post hoc $\mathrm{p}=0.016$ ) domain and a tendency toward an increase in the ANX (post hoc $\mathrm{p}=0.02$ ) and $\mathrm{PHOB}$ (post hoc $\mathrm{p}=0.026$ ) domains. Compared with the controls, the PSDI scores of both the RLS (post hoc $\mathrm{p}=0.001$ ) and PLMD (post hoc $\mathrm{p}=0.002$ ) patients were higher, and the GSI and PSTT scores were higher only in those with RLS (post hoc $\mathrm{p}=$ 0.001 each) (Fig. 1). The differences in the SOM T-score between the RLS and PLMD groups remained significant after adjusting for age, PSQI, ISI, BDI-II and PLMI (adjusted p-value $=0.029$ ).

After adjusting for age, significant correlations were found between the PLMI and O-C $(\mathrm{r}=0.321, \mathrm{p}=0.046)$, DEP $(\mathrm{r}=$ $0.420, p=0.008), \operatorname{ANX}(r=0.335, p=0.037), \operatorname{PHOB}(r=0.547, p$ $<0.001)$, PSY $(\mathrm{r}=0.385, \mathrm{p}=0.015)$, and GSI $(\mathrm{r}=0.424, \mathrm{p}=0.007)$ domain scores. Only PHOB was correlated with the PLMAI ( $\mathrm{r}$ $=0.596, \mathrm{p}<0.001)$.

\section{DISCUSSION}

We conducted systematic psychosomatic evaluations using the SCL-90-R in patients with PLMD and RLS. Psychosomatic distress was greater in the RLS patients and lesser in the PLMD patients. The patients with RLS showed higher SCL-90-R scores in the SOM, O-C, I-S, ANX, and PSY domains compared to the controls. The PLMD patients only showed higher SCL-90-R scores in the HOS domain compared to the controls. Moreover, the RLS patients had higher SOM scores than the PLMD patients after adjusting for age, PSQI, ISI, and PLMI.

The SCL-90-R T-scores in the SOM, O-C, I-S, ANX, and PSY domains were significantly higher in the patients with RLS. RLS is well known to be associated with a higher risk of depression and anxiety [10,24,25]. A previous study reported elevated SCL-90-R domain scores in all but the I-S and PAR domains in RLS patients compared with healthy controls [17]; similar results were demonstrated in our study. A recent study reported similar depressive and anxiety symptoms between RLS and PLMD patients [11]. However, the patients with PLMD in our study demonstrated a higher SCL-90-R score only in the HOS domain. This difference may be due to the different demographic characteristics of the PLMD patients. The PLMD sample in our study included more males (65.0\% vs. $37.5 \%)$ and older individuals (mean age $55.0 \pm 12.0$ vs. $45.5 \pm 8.9$ ) than the previous report [11].

Compared to the PLMD and good sleepers groups, somatization was the most distinctive psychosomatic symptom in the RLS group. Furthermore, somatization was the only symptom that was significantly worse in the RLS patients than the PLMD patients. The SOM domain score has been reported to be the most significant contributing factor to psychosomatic comorbidity in RLS [17]. Somatosensory amplification involving the thalamus and limbic structures is associated with RLS [26] and patients with somatoform disorders [27,28]. Moreover, patients with somatization disorder are likely to complain of RLS symptoms. Among somatoform pain disorder patients, the prevalence of RLS is reportedly high (28-42\%) [29,30], and those with comorbid RLS suffer more prolonged and severe pain than those without RLS [30].

The PLMD and RLS patients had similar subjective sleep disturbances. Sleep disturbance is well documented in RLS patients. More than half of patients with RLS have distressing insomnia with odds ratios between 1.7 and 3.5 compared with controls [7]. The sleep disturbance is considered the reason for psychosomatic distress in RLS patients [25]. Previous studies have also reported that PLMD patients have worse insomnia symptoms [31] and impaired sleep quality than good sleepers [11], which is consistent with our findings in this study. The PLMD patients exhibited sleep disturbance on PSG and in-

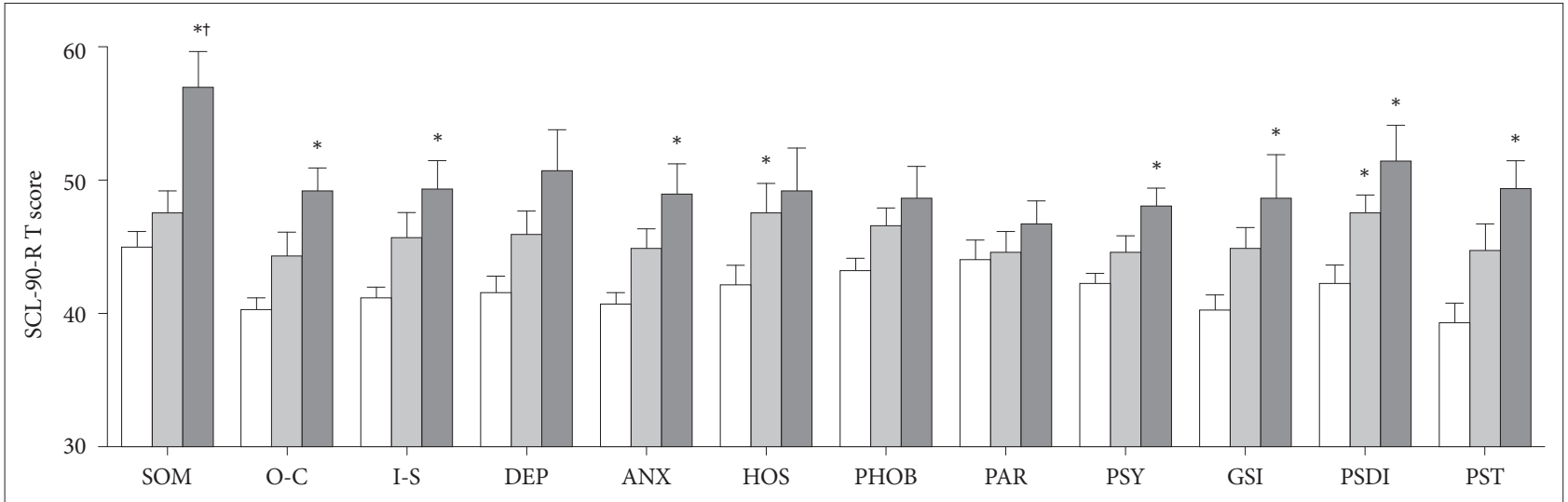

Fig. 1. SCL-90-R T-scores of the good sleeper controls and the PLMD and RLS patients. *Post hoc $p<0.017$ compared with the control group. ${ }^{\dagger}$ Post hoc $p<0.017$ compared with the PLMD group; Mann-Whitney U test. SCL-90-R: Symptom Checklist 90-Revised, PLMD: periodic limb movement disorder, RLS: restless legs syndrome, SOM: somatization, O-C: obsession-compulsion, I-S: interpersonal sensitivity, DEP: depression, ANX: anxiety, HOS: hostility, PHOB: phobic anxiety, PAR: paranoid ideation, PSY: psychoticism, GSI: Global Severity Index, PSDI: Positive Symptom Distress Index, PST: Positive Symptoms Total. 
somnia symptoms similar to those in the patients with RLS. Our results suggest that mood disorders in RLS and PLMD patients have a more complex etiology than simply a sleep disturbance or dopaminergic dysregulation. This hypothesis is consistent with previous studies reporting an independent association between comorbid psychological distress and RLS regardless of sleep disturbances [32,33].

PLM during sleep was associated with increased severity in several psychosomatic domains, such as O-C, DEP, ANX, PHOB, and PSY. PLM is associated with sleep disturbance and considered to be linked to dopamine dysfunction [34], which may explain the associated psychosomatic distress. PLM may enhance cortical and autonomic arousal during sleep [35,36]. Similarly, in our study, the PLMI was associated with the arousal index. A population-based cohort study showed an association between PLMI > 15/hr and subjective and objective sleep disturbance [37,38], which is consistent with our study.

The results of this study should be interpreted with caution considering its limitations. Number of patients included was small, and although we used nonparametric statistics for the analysis, type 1 error cannot be excluded. Because this study adopted a cross-sectional design, evaluating any causal relationship between neuropsychiatric distress and RLS or PLMD is challenging. Although we only included patients without any other sleep disorders, the PLMD patients were recruited from a selected population of patients with sleep disturbance. PSG was performed only in patients with RLS and PLMD and not in the good sleeper controls.

This study was the first to systematically compare neuropsychiatric symptoms in patients with PLMD and those with RLS. We found that the psychosomatic burden in the RLS patients was greater than that in the PLMD patients. Somatization was the most prominent psychosomatic symptom in the RLS group. PLMS was associated with the psychosomatic domains. Further prospective studies involving a larger number of patients evaluating psychosomatic distress in RLS and PLMD patients before and after dopaminergic treatment may clarify the relationship between psychosomatic distress and these disorders.

\section{Conflicts of Interest}

The authors have no financial conflicts of interest.

\section{Authors' Contribution}

Conceptualization: Byun JI, Shin WC. Data curation: Kim JS, Shin YY. Formal analysis: Byun JI. Methodology: Hwang KJ, Jung YJ. Supervision: Shin WC. Writing_original draft: Byun JI, Kim JS. Writing_review \& editing: Hwang KJ, Jung YJ, Shin WC.

\section{REFERENCES}

1. Earley CJ. Clinical practice. Restless legs syndrome. N Engl J Med 2003;348:2103-9.

2. American Academy of Sleep Medicine. International Classification of Sleep Disorders-3rd edition (ICSD-3). Darien (IL): American Academy of Sleep Medicine 2014.
3. Walters AS. Toward a better definition of the restless legs syndrome. The International Restless Legs Syndrome Study Group. Mov Disord 1995;10:634-42.

4. Montplaisir J, Boucher S, Poirier G, Lavigne G, Lapierre O, Lespérance P. Clinical, polysomnographic, and genetic characteristics of restless legs syndrome: a study of 133 patients diagnosed with new standard criteria. Mov Disord 1997;12:61-5.

5. Hening WA, Allen RP, Earley CJ, Picchietti DL, Silber MH; Restless Legs Syndrome Task Force of the Standards of Practice Committee of the American Academy of Sleep Medicine. An update on the dopaminergic treatment of restless legs syndrome and periodic limb movement disorder. Sleep 2004;27:560-83.

6. Moore H 4th, Winkelmann J, Lin L, Finn L, Peppard P, Mignot E. Periodic leg movements during sleep are associated with polymorphisms in BTBD9, TOX3/BC034767, MEIS1, MAP2K5/SKOR1, and PTPRD. Sleep 2014;37:1535-42.

7. Earley CJ, Silber MH. Restless legs syndrome: understanding its consequences and the need for better treatment. Sleep Med 2010;11:807-15.

8. Cho SJ, Hong JP, Hahm BJ, Jeon HJ, Chang SM, Cho MJ, et al. Restless legs syndrome in a community sample of Korean adults: prevalence, impact on quality of life, and association with DSM-IV psychiatric disorders. Sleep 2009;32:1069-76.

9. Lee HB, Hening WA, Allen RP, Kalaydjian AE, Earley CJ, Eaton WW, et al. Restless legs syndrome is associated with DSM-IV major depressive disorder and panic disorder in the community. J Neuropsychiatry Clin Neurosci 2008;20:101-5.

10. Picchietti D, Winkelman JW. Restless legs syndrome, periodic limb movements in sleep, and depression. Sleep 2005;28:891-8.

11. Hardy De Buisseret FX, Mairesse O, Newell J, Verbanck P, Neu D. While isolated periodic limb movement disorder significantly impacts sleep depth and efficiency, co-morbid restless leg syndrome mainly exacerbates perceived sleep quality. Eur Neurol 2017;77:272-80.

12. Mahowald MW. Periodic limb movements are NOT associated with disturbed sleep. Con. J Clin Sleep Med 2007;3:15-7.

13. Högl B. Periodic limb movements are associated with disturbed sleep. Pro. J Clin Sleep Med 2007;3:12-4.

14. Oksenberg A, Dynia A, Nasser K, Gadoth N. Obstructive sleep apnoea in adults: body postures and weight changes interactions. J Sleep Res 2012;21:402-9.

15. Lee HB, Song ML, Koo BB, Cho YW. Mood symptoms and restless legs syndrome without periodic limb movements during sleep: is it a clinical subtype? J Neuropsychiatry Clin Neurosci 2017;29:52-9.

16. Scholz H, Benes H, Happe S, Bengel J, Kohnen R, Hornyak M. Psychological distress of patients suffering from restless legs syndrome: a cross-sectional study. Health Qual Life Outcomes 2011;9:73.

17. Kim JB, Koo YS, Eun MY, Park KW, Jung KY. Psychosomatic symptom profiles in patients with restless legs syndrome. Sleep Breath 2013;17:1055-61.

18. Allen RP, Picchietti D, Hening WA, Trenkwalder C, Walters AS, Montplaisi J. Restless legs syndrome: diagnostic criteria, special considerations, and epidemiology. A report from the restless legs syndrome diagnosis and epidemiology workshop at the National Institutes of Health. Sleep Med 2003;4:101-19.

19. Sohn SI, Kim DH, Lee MY, Cho YW. The reliability and validity of the Korean version of the Pittsburgh Sleep Quality Index. Sleep Breath 2012;16:803-12.

20. Cho YW, Lee JH, Son HK, Lee SH, Shin C, Johns MW. The reliability and validity of the Korean version of the Epworth sleepiness scale. Sleep Breath 2011;15:377-84.

21. Cho YW, Song ML, Morin CM. Validation of a Korean version of the insomnia severity index. J Clin Neurol 2014;10:210-5.

22. Jo SA, Park MH, Jo I, Ryu SH, Han C. Usefulness of Beck Depression Inventory (BDI) in the Korean elderly population. Int J Geriatr Psychiatry 2007;22:218-23.

23. Kim JH, Kim GI. The standardization study of symptom checklist- 
90-revision in Korea III. Ment Health Res 1984;2:278-311.

24. Hornyak M. Depressive disorders in restless legs syndrome: epidemiology, pathophysiology and management. CNS Drugs 2010;24:89-98.

25. Becker PM, Sharon D. Mood disorders in restless legs syndrome (Willis-Ekbom disease). J Clin Psychiatry 2014;75:e679-94.

26. Rizzo G, Li X, Galantucci S, Filippi M, Cho YW. Brain imaging and networks in restless legs syndrome. Sleep Med 2017;31:39-48.

27. Perez DL, Barsky AJ, Vago DR, Baslet G, Silbersweig DA. A neural circuit framework for somatosensory amplification in somatoform disorders. J Neuropsychiatry Clin Neurosci 2015;27:e40-50.

28. Lemche E, Giampietro VP, Brammer MJ, Surguladze SA, Williams SC, Phillips ML. Somatization severity associated with postero-medial complex structures. Sci Rep 2013;3:1032.

29. Chatterjee SS, Mitra S, Guha P, Chakraborty K. Prevalence of restless legs syndrome in somatoform pain disorder and its effect on quality of life. J Neurosci Rural Pract 2015;6:160-4.

30. Aigner M, Prause W, Freidl M, Weiss M, Izadi S, Bach M, et al. High prevalence of restless legs syndrome in somatoform pain disorder. Eur Arch Psychiatry Clin Neurosci 2007;257:54-7.

31. Ancoli-Israel S, Kripke DF, Klauber MR, Mason WJ, Fell R, Kaplan O. Periodic limb movements in sleep in community-dwelling elderly.
Sleep 1991;14:496-500.

32. Li Y, Mirzaei F, O’Reilly EJ, Winkelman J, Malhotra A, Okereke OI, et al. Prospective study of restless legs syndrome and risk of depression in women. Am J Epidemiol 2012;176:279-88.

33. Szentkiralyi A, Molnar MZ, Czira ME, Deak G, Lindner AV, Szeifert L, et al. Association between restless legs syndrome and depression in patients with chronic kidney disease. J Psychosom Res 2009;67:173-80.

34. Karatas M. Restless legs syndrome and periodic limb movements during sleep: diagnosis and treatment. Neurologist 2007;13:294-301.

35. Sieminski M, Pyrzowski J, Partinen M. Periodic limb movements in sleep are followed by increases in EEG activity, blood pressure, and heart rate during sleep. Sleep Breath 2017;21:497-503.

36. Sforza E, Pichot V, Barthelemy JC, Haba-Rubio J, Roche F. Cardiovascular variability during periodic leg movements: a spectral analysis approach. Clin Neurophysiol 2005;116:1096-104.

37. Hornyak M, Feige B, Riemann D, Voderholzer U. Periodic leg movements in sleep and periodic limb movement disorder: prevalence, clinical significance and treatment. Sleep Med Rev 2006;10:169-77.

38. Haba-Rubio J, Marti-Soler H, Tobback N, Andries D, Marques-Vidal P, Vollenweider P, et al. Clinical significance of periodic limb movements during sleep: the HypnoLaus study. Sleep Med 2018;41:45-50. 